\title{
Electronic Structure of Ternary Antimonides GdNiSb
}

\author{
X.-P. Wei, X.-R. Hu, Y.-C. Dai, T. Lei, S.-B. Chu and J.-B. Deng* \\ Department of Physics, Lanzhou University, Lanzhou 730000 \\ People's Republic of China
}

(Received March 29, 2010; revised version September 26, 2010; in final form October 9, 2010)

\begin{abstract}
In the paper density functional theory method was applied to explore the electronic and magnetic properties of the GdNiSb in low-temperature phase with cubic MgAsAg-type structure and in the high-temperature phase. The calculations were performed by first principles full-relativistic full potential local orbital method within the local spin density approximation. The calculations results show the metallic character of GdNiSb compound in the high-temperature phase with hexagonal $\mathrm{AlB}_{2}$-type structure. For the low-temperature phase of the cubic GdNiSb system, they indicate a semiconducting behavior. The density of states below the Fermi level is greater in high-temperature phase than in low-temperature one, the calculated magnetic moment is in good agreement with an available experimental value.
\end{abstract}

PACS: 71.20.-b, 71.15.Ap, 71.15.Mb, 71.20.Eh, 71.20.Lp

\section{Introduction}

Intermetallic compounds that have attracted a great deal of attention during the last years are known as LnMX, where Ln is a lanthanide element, $\mathrm{M}$ is a transition metal and $\mathrm{X}$ is an $s p$-electron element. These compounds offer a large variety of structure types $[1,2]$. Among this family there is focus on electronic, transport and magnetic properties such as fermionic [3, 4], heavy-fermion and half-metallic behaviors in some Ce compounds $[5,6]$, giant magnetoresistance in heavy rare-earth compounds [7-9]. These ternary compounds of

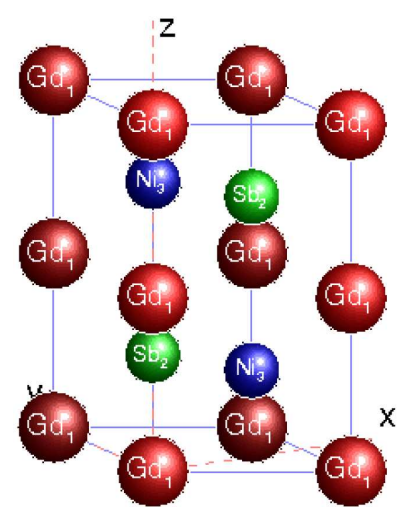

Fig. 1. The $\mathrm{AlB}_{2}$-type crystal structure of the high-temperature phase.

* corresponding author; e-mail: dengjb@lzu.edu.cn

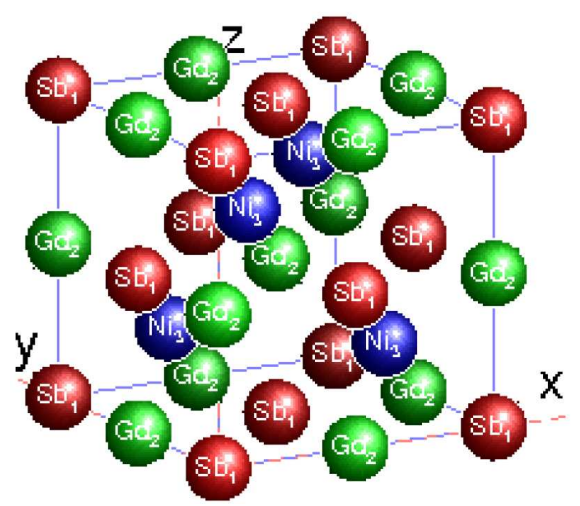

Fig. 2. The MgAgAs-type crystal structure of the low-temperature phase.

rare earths have been studied intensively in recent years [10-15].

In this paper, the electronic structure of GdNiSb compound has been studied using the local spin density approximation (LSDA) implemented in the full potential local orbital (FPLO 9.00) [16-18]. GdNiSb compound crystallizes in an ordered variant of the $\mathrm{AlB}_{2}$ structure. Here the nickel and antimony atoms are ordered on the $\mathrm{B}$ position of the $\mathrm{AlB}_{2}$ structure, resulting in a doubled $c$ axis in Fig. 1 (ZrBeSi-type structure, P63/mmc, No. 194) for HT phase and in MgAgAs-type structure for LT phase $(F \overline{4} 3 m$, No. 216), as is shown in Fig. 2. We used the Wyckoff positions and the lattice constants measured by the single-crystal X-ray diffraction method [19, 20]. The band calculations were performed for two phases based 
on the experimetal lattice parameters taking into account their potential applications in magnetic sensors, magnetic random access memories (MRAM) and spintronics [21], the rare-earth compounds are widely considered to be very promising materials.

\section{Method of calculations}

In order to study electronic structure of the ferromagnetic GdNiSb compound, we used the FPLO-9.00 code $[16,17]$. The LSDA exchange-correlation potential was used in the Perdew and Wang form [22]. The full-relativistic self-consistent calculations were performed for $14 \times 14 \times 14 k$-mesh which corresponds to $192 k$-points in the irreducible Brillouin zone. Computations of band structure and electronic densities of states (DOS) were done with spin-polarization and default initial spin splitting. The self-consistent criterion was equal to $10^{-8} \mathrm{Ha}$ for the total energy.

\section{Results and discussions}

We present results of full-relativistic band structure calculations based on the first principles. The plots of the band structure of HT and LT GdNiSb compound are presented in Figs. 3 and 4, respectively. The metallic ground state of the hexagonal GdNiSb was confirmed in contrast to the semiconducting behavior found in the cubic GdNiSb compound.

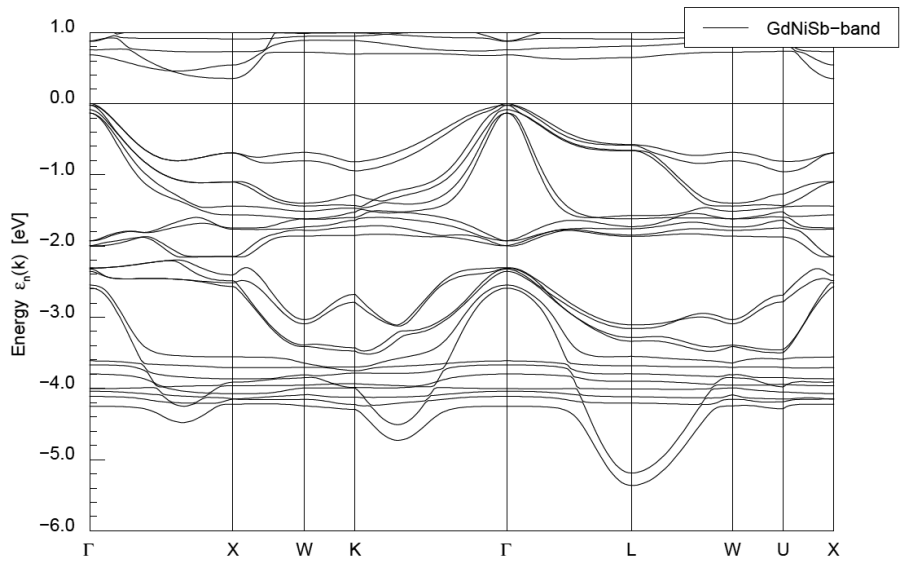

Fig. 3. Band structure of the ferromagnetic LT-GdNiSb compound.

The total and partial DOS per unit cell are presented in Figs. 5 and 6 for HT and LT phases with separated spin-up and spin-down channels. The contribution from $\mathrm{Gd}, \mathrm{Ni}$, and $\mathrm{Sb}$ atoms to the total DOS were also given. For HT and LT phase structure, the main peaks are located near $-5 \mathrm{eV}<E<1 \mathrm{eV}$ and $-5 \mathrm{eV}<E<1.5 \mathrm{eV}$, containing the contribution of antibonding bands formed by $4 f$ states of $\mathrm{Gd}$ for both phases. The bonding-band peaks located between -2 and $-0.5 \mathrm{eV}$ are ascribed to $3 d$ states of $\mathrm{Ni}$ atom. For $\mathrm{HT}$ phase structure, the total

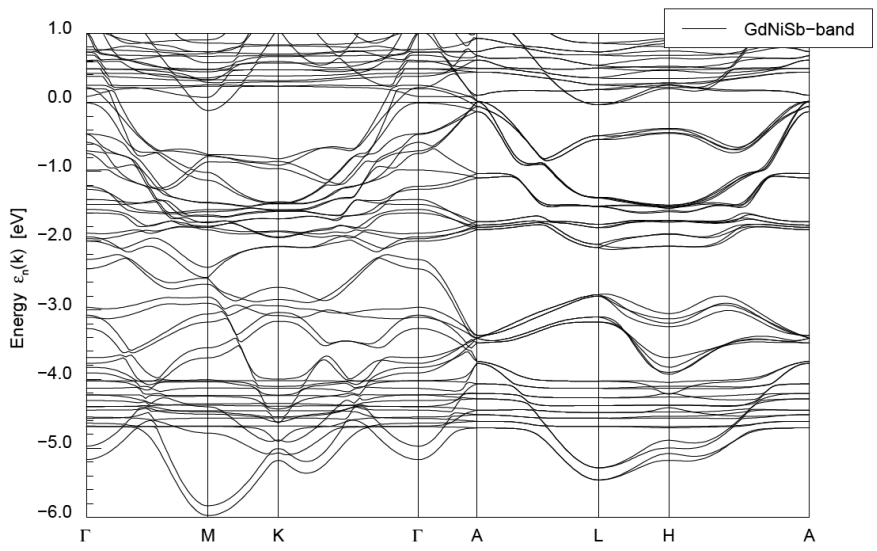

Fig. 4. Band structure of the ferromagnetic HT-GdNiSb compound.

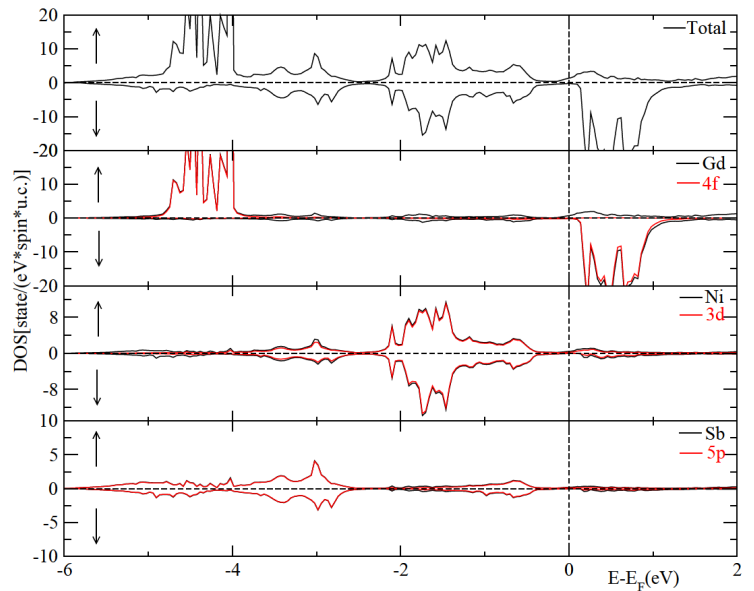

Fig. 5. The total and partial density of states for HT phase of the ferromagnetic GdNiSb compound. The arrows $\uparrow$ and $\downarrow$ assign spin-up and spin-down states. The zero energy value corresponds to the Fermi level $\left(E_{\mathrm{F}}\right)$.

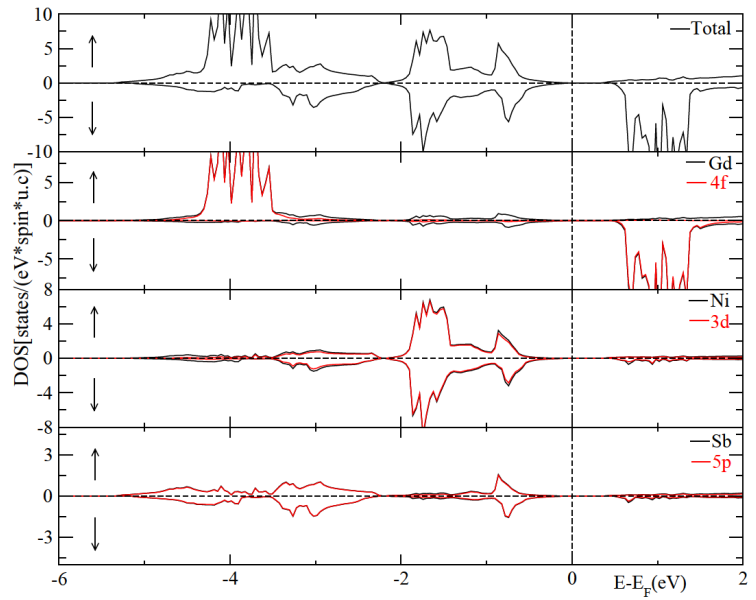

Fig. 6. The same as in Fig. 5 but for LT phase of the ferromagnetic GdNiSb. 
TABLE

Orbital and spin magnetic moments for Gd atom, total magnetic moment and experimental value in $\mu_{\mathrm{B}}$ for $\mathrm{AlB}_{2}$ and MgAgAs-type structures ( $\mu_{\exp }$ cited in Ref. [19]).

\begin{tabular}{c|c|c|c|c}
\hline \hline Type & $M_{\mathrm{O}}^{\mathrm{Gd}}$ & $M_{\mathrm{S}}^{\mathrm{Gd}}$ & Total & $\mu_{\exp }$ \\
\hline MgAgAs-type & 0.05 & 7.01 & 7.00 & $8.1(5)$ \\
$\mathrm{AlB}_{2}$-type & 0.10 & 6.99 & 14.02 & -
\end{tabular}

DOS at the Fermi level is equal to zero and close to zero (1.58 states $/ \mathrm{eV}$ ) for LT- and HT-phase structures, respectively. Although the position and width of peaks are modified partially, both LT and HT phases are quite similar as to the shape of the total DOS. The density $4 f(3 d)$ states of $\mathrm{Gd}(\mathrm{Ni})$ atom of HT phase at the Fermi level has slightly higher value (within the numerical accuracy of the code) than that of LT structure.

We also show the magnetic moment in Table, the result shows that theoretical values are in good agreement with an available experimental value.

\section{Acknowledgments}

The authors would like to thank Deepa Kasinathan, Sushil Auluck, Wen-Xu Zhang and Manuel Richter for many useful discussions.

\section{References}

[1] R.D. Hoffmann, R. Pöttgen, Z. Kristallogr. 216, 127 (2001).

[2] R. Pöttgen, D. Johrendt, Chem. Mater. 12, 875 (2000).

[3] G. Seyfarth, J.P. Brison, G. Knebel, D. Aoki, G. Lapertot, J. Flouquet, Phys. Rev. Lett. 101, 046401 (2008).

[4] K. Sun, H. Yao, E. Fradkin, S.A. Kivelson, Phys. Rev. Lett. 103, 046811 (2009).

[5] H. Fujii, Y. Uwatoko, M. Akayama, K. Satoh, Y. Maeno, T. Fujita, J. Sakurai, H. Kanimura, T. Okamoto, J. Appl. Phys. (Suppl.) 26, 549 (1987).
[6] A. Slebarski, W. Glogowski, A. Jezierski, A. Czopnik, A. Zygmunt, Phys. Rev. B 70, 184429 (2004).

[7] F. Casper, C. Felser, Solid State Commun. 148, 175 (2008).

[8] P. Siffalovic, E. Majkova, L. Chitu, Y. Halahovets, M. Jergel, R. Senderak, S. Luby, M. Weis, A. Satka, B. Szymański, F. Stobiecki, A. Timmann, S.V. Roth, Acta Phys. Pol. A 115, 332 (2009).

[9] P. Kumar, N.K. Singh, K.G. Suresh, A.K. Nigam, S.K. Malik, J. Appl. Phys. 101, 013908 (2007).

[10] P. Daniel, P. Javorský, J. Prchal, E. Šantavá, S. Daniš, Acta Phys. Pol. A 113, 331 (2008).

[11] L. Gondek, A. Szytula, O. Prokhnenko, Acta Phys. Pol. A 113, 1179 (2008).

[12] M. Hermanowicz, A. Jezierski, J. Kaczkowski, D. Kaczorowski, Acta Phys. Pol. A 115, 226 (2009).

[13] F. Casper, C. Felser, R. Seshadri, C. Peter Sebastian, R. Pöttgen, J. Phys. D, Appl. Phys. 41, 035002 (2008).

[14] F. Casper, H. Chandra Kandpal, G.H. Fecher, C. Felser, J. Phys. D, Appl. Phys. 40, 3024 (2007).

[15] M. Jirsa, M. Rameš, M. Muralidhar, Acta Phys. Pol. A 113, 223 (2008).

[16] I. Opahle, K. Koepernik, H. Eschrig, Phys. Rev. B 60, 14035 (1999).

[17] K. Koepernik, B. Velicky, R. Hayn, H. Eschrig, Phys. Rev. B 55, 5717 (1997).

[18] K. Koepernik, H. Eschrig, Phys. Rev. B 59, 1743 (1999).

[19] K. Hartjes, W. Jeitschko, J. Alloys Comp. 226, 81 (1995).

[20] V.K. Pecharskii, Y.V. Pankevich, O.I. Bodak, Sov. Phys.-Crystallogr. 28, 97 (1983).

[21] C. Felser, G.H. Fecher, B. Balke, Angew. Chem. Int. Ed. 46, 668 (2007).

[22] J.P. Perdew, Y. Wang, Phys. Rev. B 45, 13244 (1992). 